\title{
Secure the Data by using Image Compression in Multi-Cloud Storage
}

\author{
Menaka. S, Rohini. S
}

\begin{abstract}
Cloud storage providers are in ability for trust the data available and accessible that makes physical condition to be secure and running. Organizations bargain storage capacity from the providers to store user data, business, or request data. Clients desires to furnish their data to public cloud servers (PCSs). Recently got security troubles take to solve and aid more client's improvement their records in multi-cloud Storage. The customers allow saving information in the cloud the use of services supplied by using more than one cloud storage companies (CSPs). It is a hopeful method to enlarge the degree of records availability and confidentiality, as all at once these distinctive CSPs are out of provider at the identical time or collude with each other to extract records of a user. This suggest a survey on the viable protection merits by means of creating use of multiple wonderful clouds at the identical time. In addition, this advice work as merging and splitting standards at some point of storage in cloud environment. In file access, personal keys produced the use of digital key generator. 3DES encryption algorithm generates key in a cipher text format to the users. Towards facts confidentiality, this advocate an assured facts hiding and spitting picture technique in cloud storage. In propose work, essential purpose will be photo compression with mutable statistics hiding approach whilst storing in the real cloud for data hiding \& photo compression via the use of Discrete Wavelet Transform (DWT) algorithm.
\end{abstract}

Keywords: Cloud Computing, Identity-Based Cryptography, Remote Data Integrity, Proxy Public Key Cryptography Checking.

\section{INTRODUCTION}

The fast development of manipulative and communication techniques, a good deal of information generated. This huge information wants an allocation of computation resource and bigger space for storing. Over the previous years, cloud computing satisfies the pertaining to requirements and develops rapidly [1]. It takes the facts procedure as a deal, like calculating, loading records security, etc. By mistreatment lot of frequent cloud platform reachable as public, purchaser's region unit mitigated of the burden for storage management, widely wide-spread facts get right of entry to with freelance geographical locations, etc. Thus, greater purchasers would similar to grant their records by means of mistreatment a remote cloud laptop gadget [2]. In open cloud the consumer store, their huge records inside the faraway public cloud severs. Since the facts kept outside of the management of the purchasers, it entails the security dangers in relationships of reliability, secrecy and comfort of information.
Remote records integrity checking could be a primitive, which might also be accustomed to convert the cloud customers that their facts vicinity is unit unbroken intact. In some cases, the information owner (IO) could also be managed to get admission to the common public cloud sever; the IO can supply the project of records process and uploading to the third party, for instance of the proxy. On the opposite facet, the remote statistics integrity checking protocol ought to be reasonable to create it fabulous for capacity-limited finish units [3].Thus, focus on identitybased public cryptography and proxy public key cryptography; The Proposed machine of the clients transfers their information to PCS and shape their far-off data's integrity by means of web. Once the client is a reserved administrator, about sensible issues can occur. If the administrator suspected of being worried into the industrial fraud, police is abstracting him. Throughout the duration of investigation, the manager network to defend against collusion controls the price. However, the manager's legal commercial enterprise can continue all through the length of investigation. Once an outsized of statistics is generated, UN corporation will facilitate him for these information? If these statistics cannot be processing in time, the manager can face the loss of financial interest. To stop these cases happening, the manager ought to delegate the proxy to method its information, as an example, of his non-public assistant. However, the manager can do far-flung data integrity checking. Public checking can acquire some hazard of unseaworthy privations [5]. As an example, the stored information can also be recognized by way of the cruel verifiers .Once the uploaded records is confidential, onpublic far flung records reliability proving is critical. Though the secretary has the authority to switch the records for the manager, he/she nonetheless can't test the manager's remote statistics integrity until the supervisor councils him. In PKI (public key infrastructure), far-off facts reliability checking protocol can function by way of certificates administration [6]. Once the manager delegates some entities to perform the remote facts integrity checking, it will assessments the generation, renewals, delivery, certificates verification, revocation etc. In public cloud computing, low computation functionality acquired by these devices, iPad, like portable, etc. ID-PKC will eliminate the state-of-the-art certificate management. Therefore, to enlarge the strength, identity built proxy oriented statistics uploading and far-off statistics integrity checking is greater convinces. Thus, it will be quintessential to overview the [13] protocol.

Rohini. S, Assistant Professor, Department of Computer Science and Engineering, T.J.S. Engineering College, Peruvoyal. 


\section{LITERATURE REVIEW}

[4] Implemented a concept of data hiding and image transformation using cloud storage. In this, they proposed Secure Reversible Data Hiding videos storage is more secure in cloud. The uploaded video can generate the video frames; encrypted frames then transformed into image. That occurred in the user viewpoint. That transformed frame available in cloud. These frames contains the embedded image as watermarked images. Additional data in the frames erased by user and get encrypted image, while decryption usual frames has generated from cloud.

[11] Proposed safety for multi-cloud storage of records splitting with dynamic technique. They have deal with that cloud computing has swiftly elevated; cloud computing safety remains taken into consideration the essential problem inside the cloud to determine the surroundings. They proposed a secured facts garage in cloud, each consumer with a higher data storage with the exceptional Qos (Security and availability of facts) presented via available cloud service companies. By splitting and meting out client's records, model has furnished its expertise of offering a client with open storage. The paper model is effective in data as a provider, which could prolonged in added package deal prototypes of cloud.

[12] have tended it also has provision the index based totally cryptographic information reducing in Multi-Cloud storage offerings to reduce the document merging conflicts and on call for value for the clients. It make clients better and fair possibilities for choice making system to pick multicloud garage services for steady sharing of statistics primarily based on take into account. The proposed artwork guarantees that report cutting created at the kind of storage services. More than 4 cloud garage services is used for confidentiality and now not something on the Cloud Storage Service Providers can repair critical facts from the portions of data stored on its servers, without getting some greater bits of statistics from other storage carrier vendors. In our technique, it presume that all contributing garage cloud carrier companies, consisting of Drop Box ,Google Drive or other CPs, have a common hobby on securing the infrastructure and records in opposition to out of doors, 0.33 birthday party adversaries. When placing via them in present gadget conditions .This artwork recognizes those duties, however recollect them out of the opportunity of our modern-day-to-day work.

[9] Spoke to Confident Cloud Storage in Vertical Partitioning Algorithm. The vertical dividing calculation utilized to secure the information in an effective way. The java stage executed calculations and results are contrasted and different calculations.

[10] Proposed multi-distributed storage framework utilizing circulated record framework. In this exploration article, encryption, parting of client information and putting away in different cloud servers are been tended to. Likewise correlation of capacity between single cloud and different mists are been expounded.

\section{EXISTING SYSTEM}

Because of cloud system migration, more number of clients move their information to open cloud servers (PCSs).
Lot of security problems are resolved to gain the confidentiality of the client. When the clients authorized and restricted, then try to use the data using proxy server. That cause the security problems play an important role in the common cloud systems. In addition, the admin should create assured the client-outsourced data is secure [7]. That results no complete solution for security and reliability. Example: If the intermediary user guess the key of a registered users file or data, the intermediary user will find the file easily.

\section{PROPOSED SYSTEM}

The propose analysis on the possible safety merits by creating use of various different clouds simultaneously. In addition, to this center of attention on splitting and merging ideas during storage in multi-cloud environment. In file access, digital key generates the key. This protects the records with consistency and protected in more than one clouds in user economy. To grant statistics privacy that focus on a secure image compression and hiding the data technique in cloud storage. In proposed work focus on image compression with information hiding in reversible manner while storing in the multi-cloud environment. As considering literature survey, states storage and security are the major parts of cloud storage so mainly concentrate on both image compression and information hiding in reversible manner techniques. For image compression and image data hiding, this proposes the Discrete Wavelet Transform (DWT) algorithm.

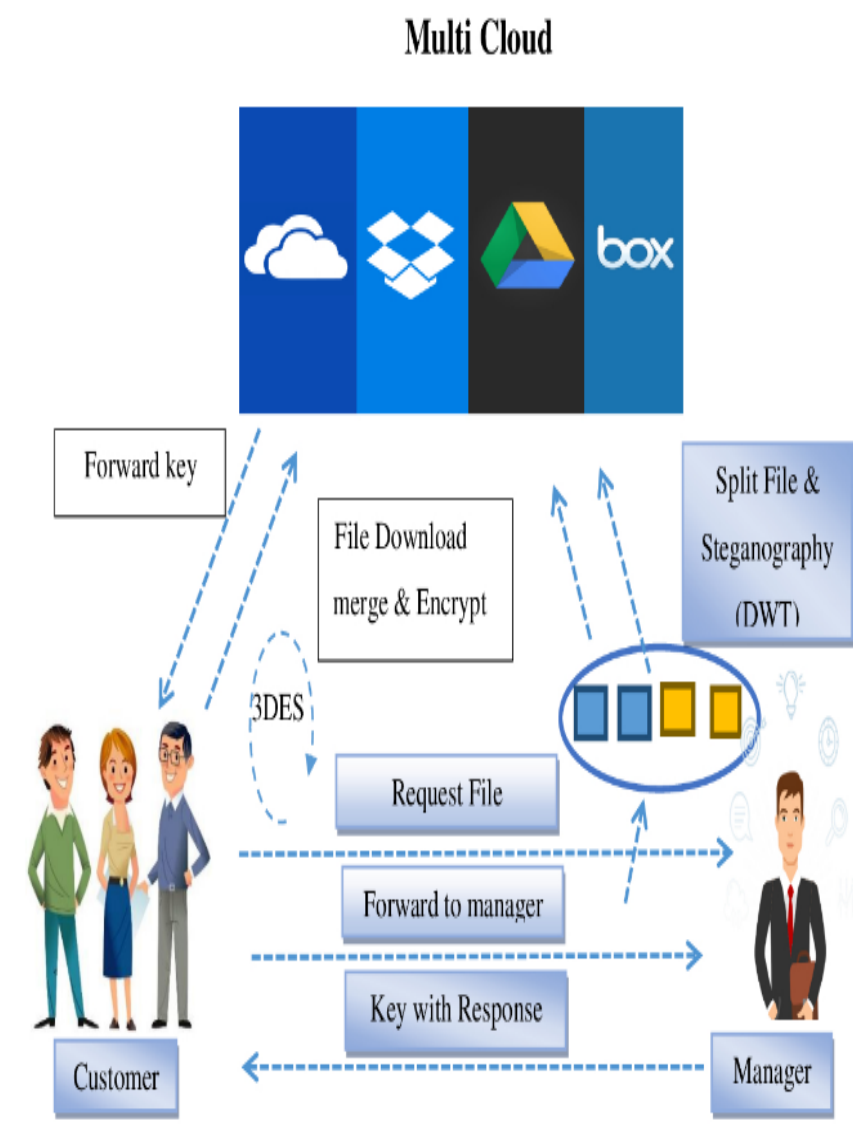

Fig. 1 System Architecture 


\section{PRELIMINARIES}

\section{Identity Management}

Initially all users must create own username and password. After Registration, the customer can log into their ID. User ID and password can be verified by this application, it checks whether already created User ID and password is matching or not matches or not in the registration from. Uncertainty it is not matches the username or password correctly then user can generate own password by using this application.

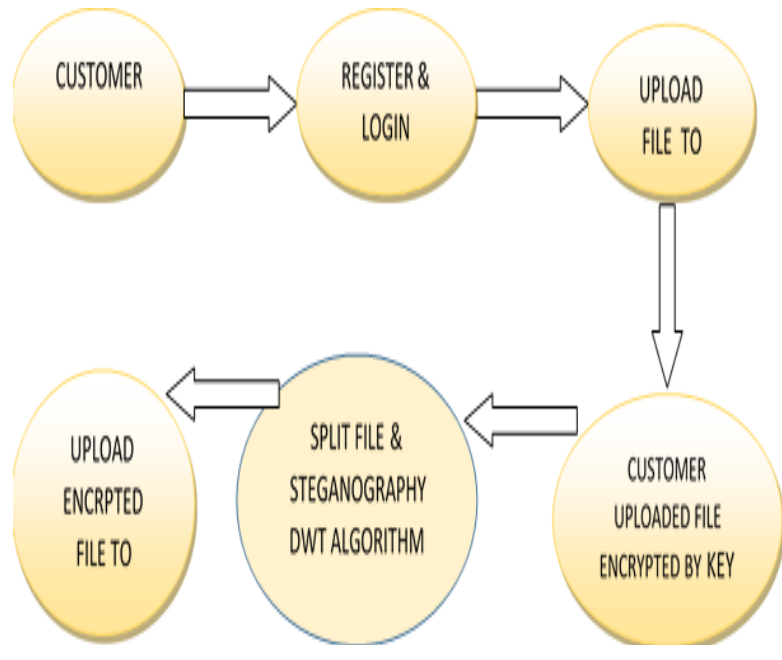

Fig. 2 Data Flow Diagram

\section{Time-based group key management:}

Time-based group Key Management rule for crypto graphical cloud storage applications, that users the proxy reencryption rule to switch principal computing venture of the cluster key management to the cloud server. Therefore, the planned TGKM theme significantly reduces the user's computation companion degreed storage overhead and makes complete usage of cloud server to obtain a systematic team key administration for the cryptographical cloud storage applications. In addition to, we tend to delivered a key seed mechanism to get a time-based dynamics team key that successfully strengths the cloud records security. Our safety find out about and overall performance analyzes each show that the planned TGKM theme may additionally be an impervious and systematic group key management protocol for the cloud storage purposes with low overheads of calculation and announcement.

\section{Spilt and Merge}

Cloud Storage normally carries business-critical statistics and processes; hence, high safety is resolution to continue strong trust relationship between the clouds customers and cloud provider suppliers. Therefore, to beat the security threats, this paper proposes more than one cloud Storage. The frequent forms of records storage likes documents and database of a chosen consumer splits and hold on inside the assorted cloud storages (e.g. Drop Box and Google Drive). Database vicinity unit to store in various cloud storages. Our software can act as a Combiner and keep very specific factors of the desk like rows as properly as columns in a number of clouds exploitation Vertical fragmentation and Horizontal fragmentation. These rows as properly as columns are going to be encrypted exploitation 3DES cryptography formula.

Throughout response, our software combines the information and sends to the admirer. Files place unit hold close in various clouds exploitation cryptographical records rending. A file splits into fragments and preserve keep of distinct cloud servers with encrypted key. Therefore, once the licensed token for the unique file is requested, searchable cryptography approves keyword locate in translated information and mix the fragments. This is regularly transferring the admirer.

\section{Triple Des:}

3DES or the Triple Data Encryption Standards Algorithm (TDEA) developed to take care of the simple flaws in DES whilst now not planning a completely new cryptosystem. Data Encryption Standard uses a 56- bit key and is unbelieved relaxed to convert refined information. 3DES is the key measurement of DES by means of pertaining to the method thrice in sequence with three diverse keys. The group key size is therefore 168-bits (3 times 56).TDEA entails the use of 3 [56-bits] DEA keys $(\mathrm{K} 1, \mathrm{~K} 2, \mathrm{~K} 3)$ in Encrypt-Decrypt-Encrypt (EDE) mode, that is plaintext is encoded with $\mathrm{K} 1$,then decrypted with $\mathrm{K} 2$,then encrypted as soon as greater with $\mathrm{K} 3$.

\section{Stegano graphy:}

Stegano graphy is science of concerning data by implanting meaning at intervals alternative, on the face of its harmless messages. Many unremarkable stegano graphy employed to supplement coding. An encoded file should hide data using stegano graphy, therefore though the encoded file is deciphered; the secret message is invisible. The aim of those methods is to cover secret information (stegano graphy) within the innocent trying carriers e.g. in standard broadcast of customers. In perfect state of affairs, third parties cannot detect hidden information exchange. For image activity and compression, we have enforced DWT algorithmic rule for economical information activity and extraction.

\section{RESULT AND DISCUSSION}

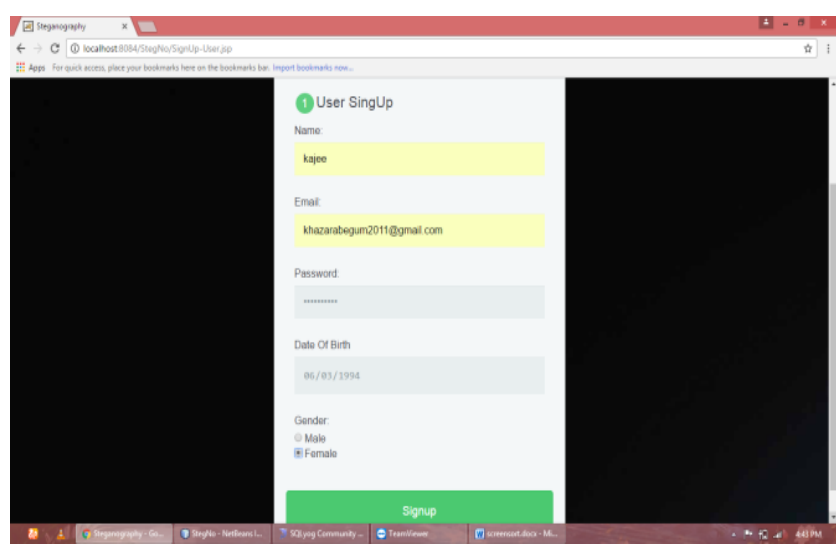

Fig. 4 User Registration Page

This work focus on the cloud server security, to verify the user details. 


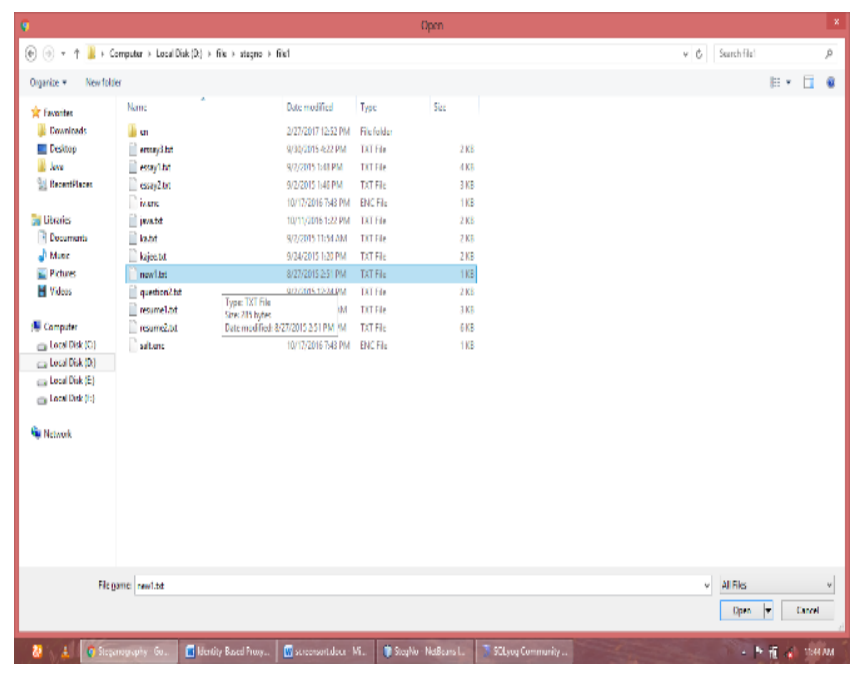

Fig. 5 File Upload Page

Once completed the file upload from the proxy server, it store the data and encrypted with the key using the algorithm.

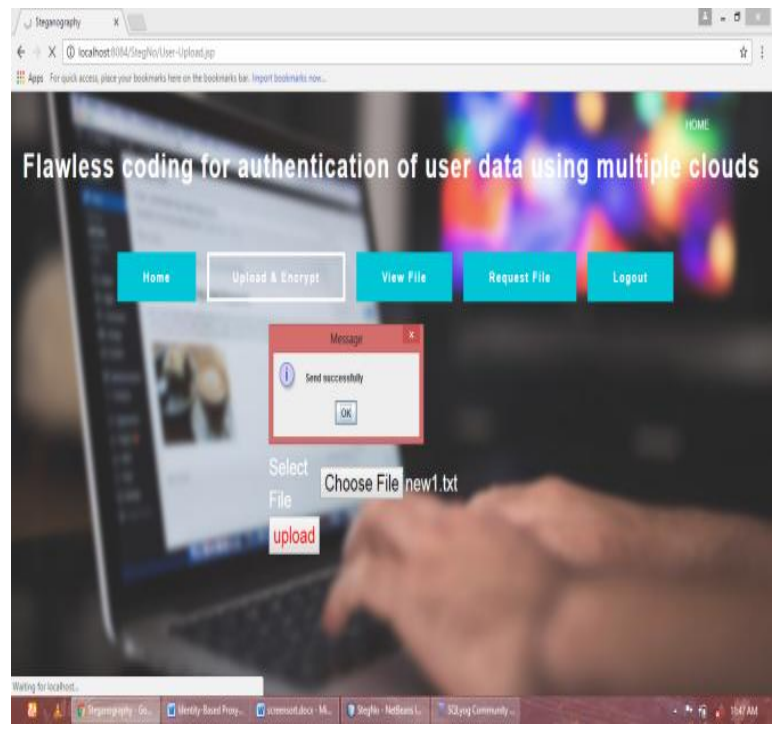

Fig. 6 Group key generated

The propose work focus on key seed mechanism is helps to strengthened the cloud security.

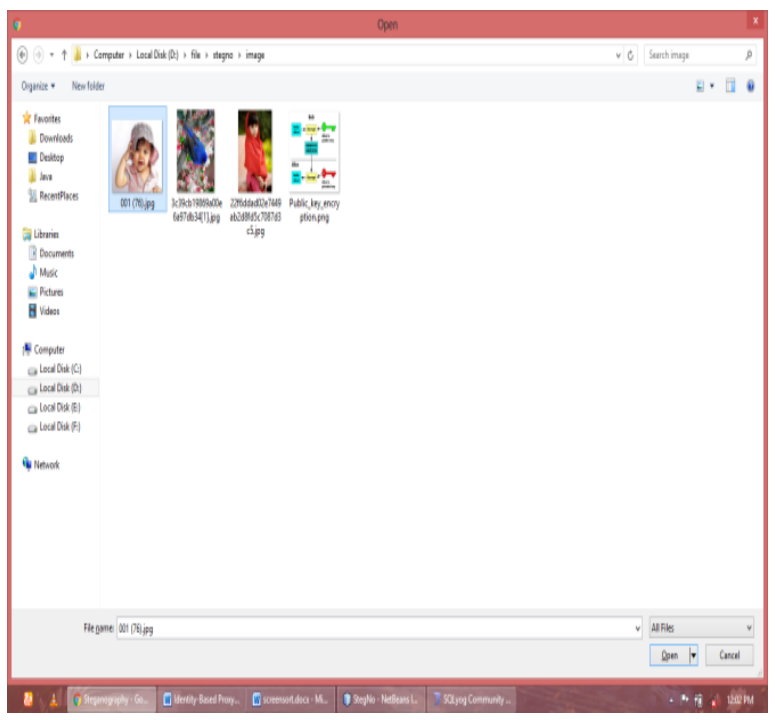

Fig. 7 Image Compression
It is converting a photograph file in such a way that it consumes much less area than the authentic file by means of information hiding (steganography) .In these situations, third parties cannot realize hidden data exchange. For photo compression, carried out DWT algorithm for environment friendly records hiding and extraction.

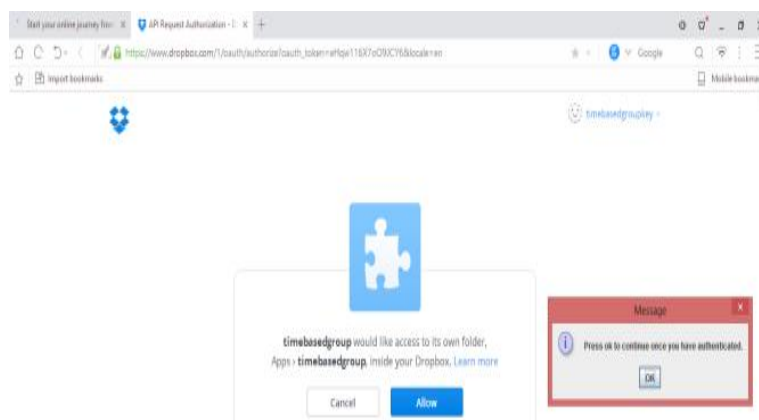

Fig. 8 File Uploaded in Cloud

The proposed work has two cloud servers namely Dropbox and CloudMe for file storage.

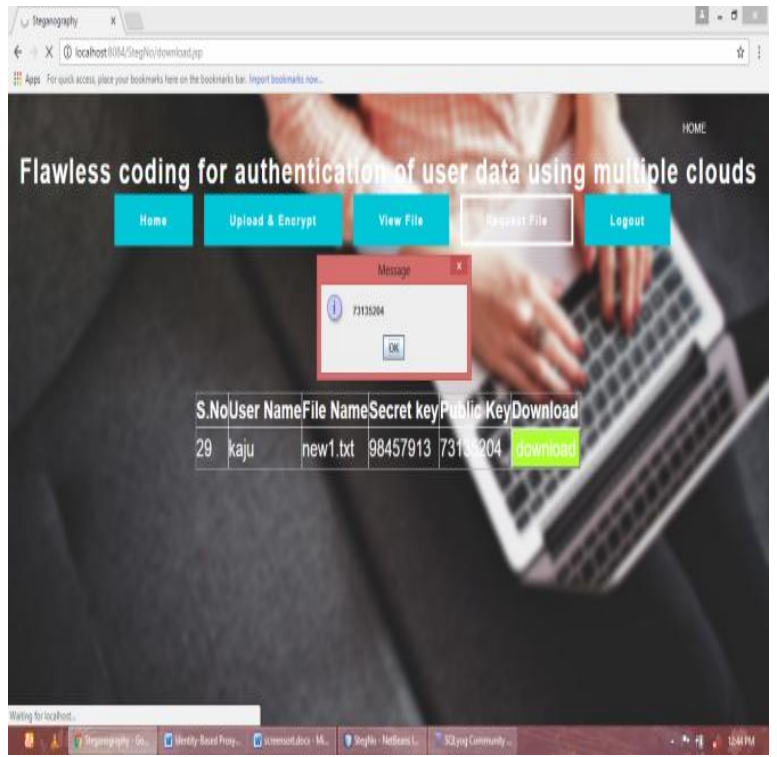

Fig. 9 File Downloaded Successfully

VII. CONCLUSION

The proposed work focus about the cloud storage security and concerned about the safety concerns, to overcome the existing system using multi-cloud with image compression and information hiding in reversible manner techniques. This work proposed information hiding in reversible manner to embedding the top-secret information in image with high security, reliability and robustness. Due to discrete algorithm, the information implanting capacity will increased in this system. Multiple data files will be loaded in cover image. 
The safety of the data's are increased because of two keys: encryption key and hiding the data key .Thus secure key generation and transmission performed by integrating virtual machines.

\section{REFERENCE}

1. Z. Fu, X. Sun, Q. Liu, L. Zhou, and J. Shu, “Achieving efficient cloud search services: Multi-keyword ranked search over encrypted cloud data supporting parallel computing," IEICE Trans. Commun., vol. E98B, no. 1, pp.190-200, 2015.

2. Y. Ren, J. Shen, J. Wang, J. Han, and S. Lee, "Mutual verifiable provable data auditing in public cloud storage," J. Internet Technol., vol. 16, no. 2, pp. 317-323, 2015.

3. M. Mambo, K. Usuda, and E. Okamoto, "Proxy signatures for delegating signing operation," in Proc. CCS, 1996, pp. 48-57.

4. Ms. Amrutha O.C and Ms. Rabina P "Secure Reversible Data Hiding Image Transformation Using Cloud Storage" IJCIR...Vol-12, No-2 (2016), pp. 193-196.

5. E.-J. Yoon, Y. Choi, and C. Kim, "New ID based proxy signature scheme with message recovery," in Grid and Pervasive Computing (Lecture Notes in Computer Science), vol. 7861. Berlin, Germany: Springer Verlag, 2013, pp.945-951.

6. B.-C. Chen and H.-T. Yeh, "Secure proxy signature schemes from the weil pairing," J. Supercomput., vol. 65, no. 2, pp. 496-506, 2013.

7. Neha, Mandeep Kaur, Enhanced Security using Hybrid Encryption Algorithm, International Journal of Innovative Research in Computer and Communication Engineering, Vol. 4, Issue 7, July 2016

8. Elham Abd Al Latif Al Badawi \& Ahmed Kayed, Survey on Enhancing The Data Security of the Cloud Computing Environment by using Data Segregation Technique, IJRRAS, 2015.

9. S. Subbiah, S. Selva Muthukumaran and T. Ramkumar, An Approach for Enhancing Secure Cloud Storage Using Vertical Partitioning Algorithm, Middle-East Journal of Scientific Research, 2015.

10. Miss. Priyanka. R. Raut, Prof. Vaidehi Baporikar, Design and Implementation of Enhanced Security In Multi cloud Storage System Using Distributed File System, IJSETR, 2015.

11. Balasaraswathi V.R.I ,Manikandan.S2 Enhanced Security for MultiCloud Storage using Cryptographic Data Splitting with Dynamic Approach, (ICACCCT) IEEE 2014, ISBN No. 978-1-4799-3914-51.

12. Dr. K. Subramanian, F. Leo John "Enhanced Security for Data Sharing in Multi Cloud Storage (SDSMC)", (IJACSA), Vol. 8, No. 3, 2017.

13. Huaqun Wang, Debiao He and Shaohua Tang, "Identity-Based ProxyOriented Data Uploading and Remote Data Integrity Checking in Public Cloud" (ID-PUIC).", IEEE,VOL..11, NO-6, JUNE 2016.

14. X. Liu, J. Ma, J. Xiong, T. Zhang, and Q. Li, "Personal health records integrity verification using attribute based proxy signature in cloud computing," in Internet and Distributed Computing Systems (Lecture Notes in Computer Science), vol. 8223. Berlin, Germany: Springer Verlag, 2013, pp. 238-251.

15. Manikanthan, S.V., Padmapriya, T., An efficient cluster head selection and routing in mobile WSN, International Journal of Interactive Mobile Technologies, 2019. 